\title{
Study of 89 Cases of Peripheral Vascular Disease by CT Angiography in a Centre of Bangladesh
}

\author{
M Faruque $^{1}$, AEMM Islam ${ }^{1}$, S Haque $^{1}$, MT Islam ${ }^{2}$, MG Kibria ${ }^{1}$, M Hossain $^{3}$, MA Salam ${ }^{1}$ \\ F Rahman ${ }^{4}$, MA Hasnat ${ }^{1}$, HILR Khan ${ }^{1}$ \\ ${ }_{-}^{1}$ Depatment of Cardiology, DMCH, Dhaka. ${ }^{2}$ Department of Radiology \& Imaging, Holy Family Red \\ Crescent Hospital, Dhaka ${ }^{3}$ Department of Vascular surgery, NICVD, Dhaka, ${ }^{4}$ Department of \\ Cardiology, BSMMU, Dhaka
}

\begin{abstract}
:
Key words: CT angiogram, Peripheral vascular disease, Risk factor

Background: The peripheral arterial disease generally refers to a disorder that obstructs the blood supply to upper and lower extremities, most commonly caused by atherosclerosis.

Objective: The purpose of this study was to observe the morphological pattern and risk factors for development of peripheral vascular disease in Bangladeshi patients suffering from peripheral vascular disease by $C T$ angiography using a multidetector scanner.

Method: Eighty nine patients with peripheral vascular disease who were referred for evaluation of peripheral vascular disease underwent CT angiography. We scanned patients from the level of the cerebral arteries to the pedal arteries in a single helical scan. CT angiograms were produced using maximum-intensity-projection, multiplanous reformation and reconstructions. Findings were graded according to nine categories: 1, normal (0\% stenosis); 2, mild (1-49\% stenosis); 3, moderate (50-74\% stenosis); 4, severe (>75\% stenosis); 5, tortuosity; 6, aneurysm, 7, calcification, 8, Arteriovenous malformation (AVM), and 9, haematoma.

Results: We found Most of the patients in our study were male (69 out of 89 patients). The mean age was $54.49 \pm 18.36$ yrs in male and $49.45 \pm 17.89$ yrs for female. Commonest risk factor in our study was hypertension $46.1 \%$, followed by diabetes $30.3 \%$, family history $27 \%$, smoking $23.6 \%$, dyslipidaemia13.5\%. Stenosis (5.61\%) was the predominate lesion followed by haematoma (4.49\%) and arterio-venous malformation (4.49\%). Abdominal aorta was mostly affected in the studied population (58.43\%) followed by Lower limb (37.08\%), Carotid (22.47\%), Renal (7.87\%) and Upper limb arteries (4.49\%).

Conclusion: CT angiography is a noninvasive technique for the imaging of peripheral vascular disease. Since no data is available from a well designed study in PVD in our country, till then the data obtained from this study can be used in Bangladesh.
\end{abstract}

(Cardiovasc. j. 2009; 1(2) : 193-200)

\section{Introduction:}

The peripheral arterial disease(PAD) generally refers to a disorder that obstructs the blood supply to upper and lower extremities, most commonly caused by atherosclerosis but may also result from thrombosis, embolism, vasculities, fibromascular dysplasia or entrapment. Where as the peripheral vascular disease includes $\mathrm{PAD}$ and other atherosclerotic conditions such as renal artery disease and carotid artery disease as well as vasculities, vasospasm, venous thrombosis, venous insufficiency and lymphatic disorder. ${ }^{1}$ Atherosclerosis is the leading cause of occlusive arterial disease of the extremities in patients over
40 years old, the highest incidence occurs in sixth and seventh decades of life. ${ }^{2}$ The pathology of PAD is similar to that of coronary artery disease. The most important risk factors are smoking, diabetes mellitus, hyperlipidaemia and hypertension. ${ }^{3}$ Tobacco increases the relative risk 2-4 times. Diabetes also increase relative risk for PAD like smoking. Among patients with PAD diabetic patients are more likely to have amputation than non diabetic patients. ${ }^{4}$ Distal disease affecting the tibial and peroneal arteries occur more frequently in diabetes. Risk of PAD increase if total cholesterol and LDL are elevated. In Framingham study it is seen that probability of

Address of Correspondence: Dr Md Faruque, Associate Professor of Cardiology, Department of Cardiology, DMCH, Dhaka, Bangladesh 
claudication in 70 years male smoker is $2.5 \%$ per 4 year, Which increases to $24 \%$ per 4 year when smoking is associated with hypertension, hypercholes trolaemia and diabetes mellitus. ${ }^{5} \mathrm{PAD}$ affects large number of people world wide but more common in USA. It is estimated that 10 million people with symptomatic disease another 20-30 million people are asymptomatic. It is estimated that $10 \%$ of people over 60 years of age are affected and prevalence continues to increase with age. The cardinal symptoms of PAD include intermittent claudication and rest pain. The location of the symptom often relates to the site of the most proximal stenosis. Buttock, hip or thigh claudication typically occurs in patients with obstruction of aorta and iliac arteries. Calf claudication characterizes femoral or popliteal artery stenosis. Ankle and pedal claudication occurs in patients with tibial and peroneal artery disease. Similarly stenosis of the subclavian, axillary or brachial arteries may cause shoulder, biceps or forearm claudication respectively. Symptoms should resolve several minutes after cessation of effort. There may be impotence in male. Rest pain in legs worse at night relieved by hanging the leg over the edge of the bed. Extremities may be cold. It may be cyanosed; there may be pain, ulceration and gangrene in 50-100 \% patients. On examination the limb is cold with dry skin and lack of hair. Pulse is diminished or absent. There may be ulceration; gangrene or dark discoloration usually starts at the toes. Death due to $\mathrm{PAD}$ is rare. Death occurs usually secondary to CAD or CVD. Relative risk of all cause of death 26 folds higher than general people. Death rate increases with decrease of ABI. Five years mortality with $\mathrm{ABI}<0.85$ is $10 \%$ where as mortality approaches $>50 \%$ when ABI is $<0.40$. Patients may be diagnosed by duplex ultrasonography, CT angiogram, MR angiogram. Conventional angiogram is gold standard for diagnosis. ${ }^{4}$

\section{Methods :}

\section{Study Population}

Peripheral C-T Angiogram was performed in 89 patients from 2006-2007 patients were referred for vascular insufficiency, severe back pain, neck swelling, uncontrolled hypertension and vertigo from outdoor patient department. This noninvasive new Technology of C-T Angiogram procedure was done on 89 patients who are suffering from peripheral vascular disease. All the procedures were done in Modern diagnostic Centre, Dhanmondi-8, Dhaka.

We scanned patients from the level of the cerebral arteries to the pedal arteries in a single helical scan. CT angiograms were produced using maximum-intensity-projection reconstructions. Findings were graded according to nine categories: 1 , normal ( $0 \%$ stenosis); 2 , mild (1-49\% stenosis); 3 , moderate (50-74\% stenosis); 4 , severe $(>75 \%$ stenosis); 5 , tortuosity; 6 , aneurysm, 7 , calcification, 8, Arteriovenous malformation (AVM), and 9, haematoma. ${ }^{6}$

Inclusion criteria:

(1) Clinically suspected peripheral vascular disease.

(2) Secondary Hypertension.

(3) Neck swelling of vascular origin.

(4) Vascular injury of Road Traffic Accident.

Exclusion criteria:

(1) Chronic renal failure.

(2) Acute pulmonary oedema (LVF).

(3) Congestive Cardiac Failure.

(4) Valvular Heart disease.

(5) Hypersensitivity to dye.

(6) Non co-operative patient ( Psychosomatic disease)

(7) Unconscious patient

\section{Procedure}

Patient preparation typically involved placing the patient supine of the CT table without a pillow or foam wedge under the knees. The rationale for not putting any support under the knees is that the field of view should be enlarged to reconstruct the arteries of interest as they move anteriorly under the pillow. Any unnecessary increase in the field-of-view was avoided as this diminishes in-plane resolution. It may additionally be advantageous to place tape around the patient's knee and ankles. The rationale for taping at the necessity to increase the field-of-view. Taping the feet helped to remind the patient to keep them immobile. Somatom Sensation 64 slice (Simens) CT angiogram machine was used. As soon as the primary CTA was completed, the reconstruction of images below the knee was prioritized and a quick assessment as 
the adequacy of these images made. If insufficient arterial opacification was present, then the second acquisition was triggered at that time. This approach assures that the pedal vessels will be adequately assessed even in the setting of substantially prolonged circulation times. When assessing the peripheral arteries, contrast medium flow rates of at least $4 \mathrm{ml}$ per second and preferably $5 \mathrm{ml}$ per second were desirable. When imaging atherosclerotic occlusive disease, the scan range was extended from the supra-renal aorta through the toes in the setting of limb threatening ischaemia or to the ankles in the setting of claudication. The presence of stenosis (considered hemodynamically significant when greater than fifty percent), occlusions, and the lengths of these abnormalities are the most important clinical observations made. Total volume of contrast administered was determined by the product of scan times and the injection rate. There were no complication related to $\mathrm{C}-\mathrm{T}$ angio and all studies are technically adequate.

The scan direction was craniocaudal for Lower Limb CTA, with the range from the level of infrarenal aorta to the pedal arch; while for Upper Limb CTA; the scan direction was caudocranial from the level of aortic arch to the palmer arch. The patients were instructed to continue quiet breathing for the duration of scan. The success of any CTA depends on the calculation of an accurate delay to start the acquisition of images after the injection of contrast, to get optimum arterial enhancement, not contaminated by the venous phase. The delay was calculated by using the test bolus technique.

\section{Data Collection \& Analysis:}

The demographic profile of all patients including age, sex was taken. The major risk factors of peripheral arterial disease i.e. diabetes, hypertension, smoking, dyslipidaemia and family history were taken in each patient. The peripheral arteries were studied- Carotid arteries, Abdominal Aorta, Upper limb arteries, Lower limb arteries and Renal arteries. Morphological pattern of lesions were described in each involved artery. Data analysis was done in SPSS programme.

\section{Results}

In all of our initial 89 patients, there has been no technical failure. The procedure has been well tolerated and in no study was there image degradation due to motion artifacts. Patients of peripheral vascular disease, with symptoms of decreased or absent pulses, claudicaton or rest pain also underwent CTA.

Table I shows age and sex distribution of the study population. It shows most of the patients were above 50yrs of age. Age and sex differences were not statistically significant.

Table II shows Mean age in male and female were $54.49 \pm 18.36$ and $49.45 \pm 17.89$ which was not significant statistically.

Table-I

Age Distribution of CT Angiography Patients(N=89)

\begin{tabular}{|c|c|c|c|c|c|}
\hline \multirow[t]{2}{*}{$\overline{\text { Age group }}$} & \multicolumn{2}{|c|}{ Sex } & \multirow[t]{2}{*}{ Total } & \multirow[t]{2}{*}{ Percentage } & \multirow{2}{*}{$\begin{array}{l}\mathrm{P} \text { value } \\
0.61 \mathrm{NS}\end{array}$} \\
\hline & Male & Female & & & \\
\hline Upto 30 & 9 & 4 & 13 & 14.6 & \\
\hline $31-40$ & 7 & 4 & 11 & 12.4 & \\
\hline $41-50$ & 9 & 1 & 10 & 11.2 & \\
\hline $51-60$ & 18 & 4 & 22 & 24.7 & \\
\hline $61-70$ & 14 & 5 & 19 & 21.3 & \\
\hline $71 \&$ above & 12 & 2 & 14 & 15.7 & \\
\hline$\overline{\text { Total }}$ & 69 & 20 & 89 & 100.0 & \\
\hline
\end{tabular}

Table-II

Mean age by sex $($ Male $=69$, Female $=20)$

\begin{tabular}{lccc}
\hline Sex & Mean & SD & P Value \\
\hline Male & 54.49 & 18.36 & NS \\
Female & 49.45 & 17.89 & \\
\hline
\end{tabular}


Table III shows distribution of risk factors in both sexes. Most of the patients were hypertensive (46.1\%) followed by diabetes (30.3\%) and smoking (23.6\%). Except smoking other risk factors did not vary significantly in two sexes.

Table-IV shows involvement of the peripheral arteries and their sex distribution. It shows abdominal aorta was the most affected artery $58.43 \%$, followed by lower limb arteries $37.08 \%$, carotid arteries $22.47 \%$, renal arteries $7.87 \%$ and upper limb arteries $4.49 \%$. Involvement of the peripheral arteries did not vary significantly in both sexes.

Table V shows morphological pattern of involved carotid arteries and their distribution in both sexes. Most commonly found lesion was stenosis (5.61\%), followed by haematoma (4.49\%), Arterio -venous malformation (4.49\%).

Table-III

Distribution of risk factors of the patients

\begin{tabular}{|c|c|c|c|c|c|}
\hline \multirow[t]{2}{*}{ Risk factors } & \multicolumn{2}{|c|}{ Positive } & \multirow[t]{2}{*}{ Total } & \multirow[t]{2}{*}{ Percentage } & \multirow[t]{2}{*}{ P Value } \\
\hline & Male & Female & & & \\
\hline Diabetes & 21 & 6 & 27 & 30.3 & NS \\
\hline Hypertension & 34 & 7 & 41 & 46.1 & NS \\
\hline Smoking & 21 & 0 & 21 & 23.6 & $\mathbf{S}$ \\
\hline Dyslipidaemia & 9 & 3 & 12 & 13.5 & NS \\
\hline Family history & 21 & 3 & 24 & 27 & NS \\
\hline
\end{tabular}

Table-IV

Distribution of the involved peripheral artery diseases by sex

\begin{tabular}{|c|c|c|c|c|c|c|c|c|}
\hline \multirow[t]{2}{*}{ Arteries involved } & \multicolumn{2}{|c|}{ Normal } & \multirow[b]{2}{*}{ Total } & \multirow[b]{2}{*}{ Percentage } & \multicolumn{2}{|c|}{ Abnormal } & \multirow[b]{2}{*}{ Total } & \multirow[b]{2}{*}{ Percentage } \\
\hline & Male & $\overline{\text { Female }}$ & & & Male & Female & & \\
\hline Carotid & 56 & 13 & 69 & 77.53 & 13 & 7 & 20 & 22.47 \\
\hline Abdominal aorta & 23 & 14 & 37 & 41.57 & 46 & 6 & 52 & 58.43 \\
\hline Upper limb & 68 & 17 & 85 & 95.51 & 1 & 3 & 4 & 4.49 \\
\hline Lower limb & 37 & 19 & 56 & 62.92 & 32 & 1 & 33 & 37.08 \\
\hline Renal & 64 & 18 & 82 & 92.13 & 5 & 2 & 7 & 7.87 \\
\hline
\end{tabular}

Table-V

Morphology Pattern of Carotid Artery by sex $(n=89)$

\begin{tabular}{lcccc}
\hline Carotid Artery & Male & Female & Total & Percentage \\
\hline Mild Stenosis & 1 & 3 & 4 & 4.49 \\
Moderate Stenosis & 1 & 0 & 1 & 1.12 \\
Tortuous & 1 & 1 & 2 & 2.25 \\
Aneurysm & 2 & 1 & 3 & 3.37 \\
Calcification & 1 & 1 & 2 & 2.25 \\
AVM & 3 & 1 & 4 & 4.49 \\
Haematoma & 4 & 0 & 4 & 4.49 \\
Normal & 56 & 13 & 69 & 77.53 \\
\hline Total & 69 & 20 & 89 & 100.00 \\
\hline
\end{tabular}




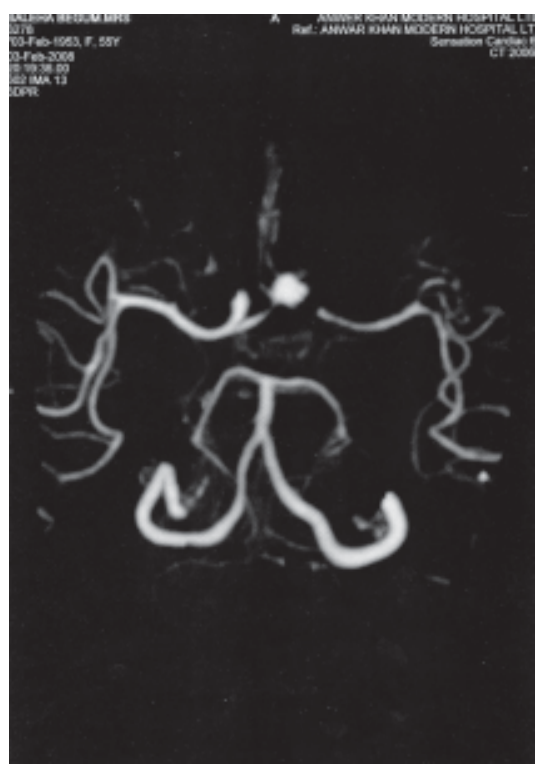

Fig.-1: Cerebral Angiogram as shown in CT

Table VI shows morphological pattern of involved abdominal aorta and their distribution in both sexes. Most commonly found lesion was stenosis (46.07), followed by calcification (6.74\%), aneurysm (3.37\%)

Table VII shows morphological pattern of involved upper limb arteries and their distribution in both sexes. Most commonly found lesion was tortuosity $(2.25 \%)$, followed by stenosis (1.12\%), aneurysm $(1.12 \%)$.

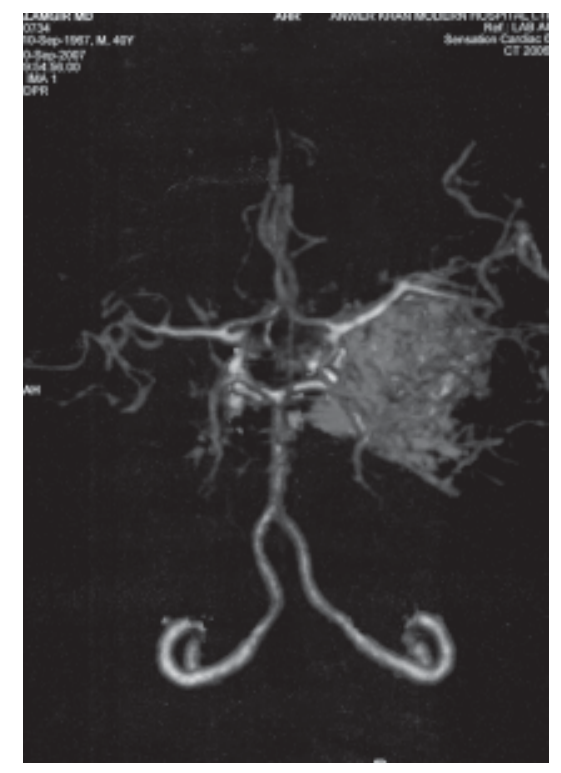

Fig.-2: Cerebral Angiogram as shown in $C T$

Table VIII shows morphological pattern of involved lower limb arteries and their distribution in both sexes. Most commonly found lesion was stenosis (5.61\%), followed by haematoma $(4.49 \%)$, Arterio venous malformation $4.49 \%$.

Table IX shows morphological pattern of involved carotid arteries and their distribution in both sexes. Most commonly found lesion was stenosis (5.61\%), followed by haematoma (4.49\%), Arterio -venous malformation $4.49 \%$.

Table VI

Morphology Pattern of Abdominal Aorta by sex $(n=89)$

\begin{tabular}{lcccc}
\hline Abdominal aorta & Male & Female & Total & Percentage \\
\hline Mild Stenosis & 4 & 2 & 6 & 6.74 \\
Moderate Stenosis & 26 & 2 & 28 & 31.46 \\
Severe Stenosis & 7 & 0 & 7 & 7.87 \\
Tortuous & 0 & 1 & 1 & 1.12 \\
Thrombus & 1 & 0 & 1 & 1.12 \\
Aneurysm & 3 & 0 & 3 & 3.37 \\
Calcification & 5 & 1 & 6 & 6.74 \\
Normal & 23 & 14 & 37 & 41.57 \\
\hline Total & 69 & 20 & 89 & 100.00 \\
\hline
\end{tabular}




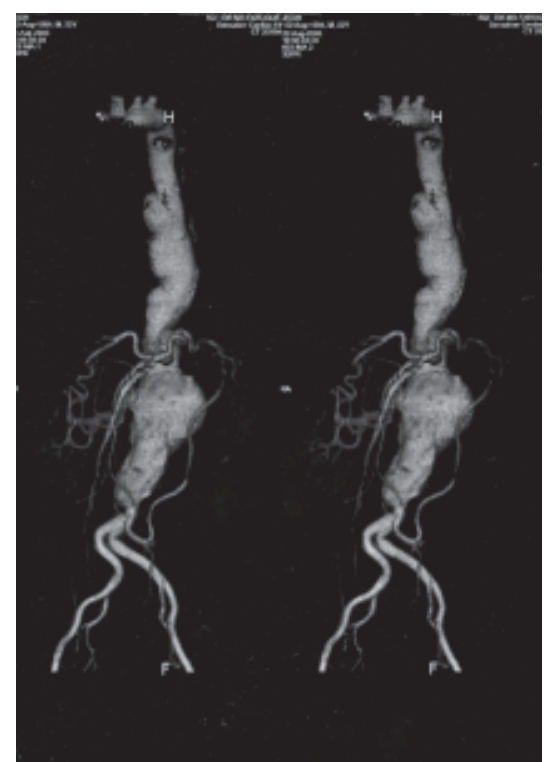

Fig: 3: Abdominal aneurysm affecting supra and infra renal region

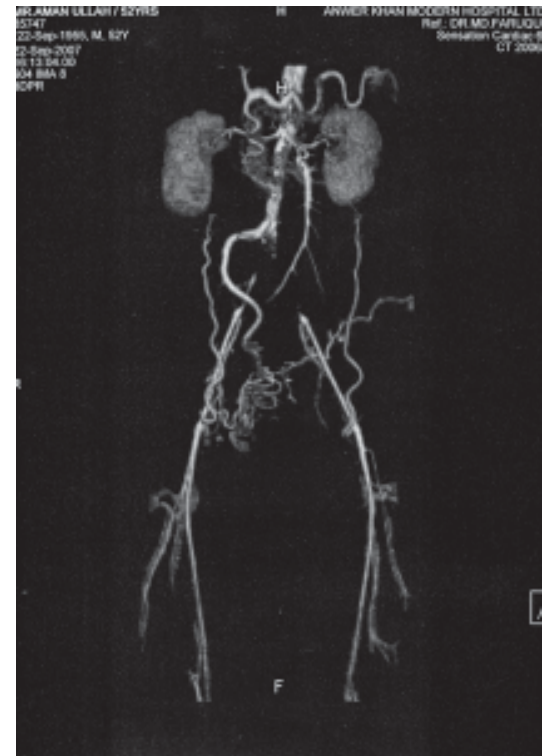

Fig: 5: PVD affecting right and left common iliac

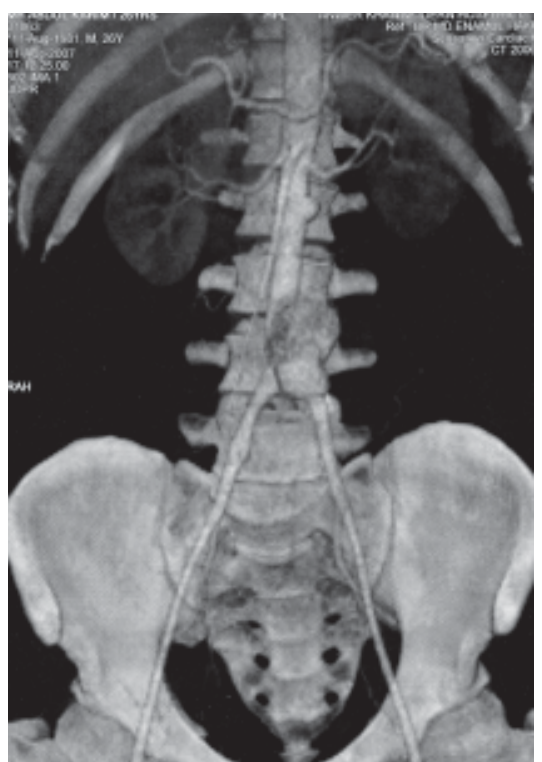

Fig: 4: Extra vascular mass compressing the lower part of the abdominal aorta near its bifurcation

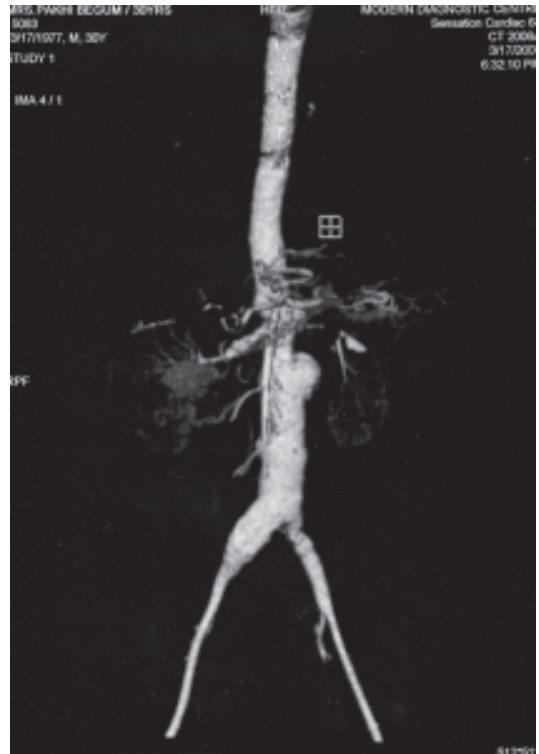

Fig: 6: Aneurysm affecting infra renal aorta and right common iliac

Table VII

Morphology Pattern of Upper Limb Artery by sex $(n=89)$

\begin{tabular}{lcccc}
\hline Upper limb artery & Male & Female & Total & Percentage \\
\hline Mild Stenosis & 0 & 1 & 1 & 1.12 \\
Moderate Stenosis & 0 & 0 & 0 & 0 \\
Severe Stenosis & 0 & 0 & 0 & 0 \\
Tortuous & 0 & 2 & 2 & 2.25 \\
Thrombus & 0 & 0 & 0 & 0 \\
Aneurysm & 1 & 0 & 1 & 1.12 \\
Calcification & 68 & 17 & 85 & 95.51 \\
Normal & 69 & 20 & 89 & 100.00 \\
\hline Total & &
\end{tabular}


Table VIII

Morphology Pattern of Lower Limb Artery by sex ( $n=89)$

\begin{tabular}{lcccc}
\hline Lower limb artery & Male & Female & Total & Percentage \\
\hline Mild Stenosis & 2 & 0 & 2 & 2.25 \\
Moderate Stenosis & 13 & 1 & 14 & 15.73 \\
Severe Stenosis & 3 & 0 & 3 & 3.37 \\
Tortuous & 0 & 0 & 0 & 0 \\
Thombus & 0 & 0 & 0 & 0 \\
Aneurysm & 0 & 0 & 0 & 0 \\
Calcification & 14 & 0 & 14 & 15.73 \\
Normal & 37 & 19 & 56 & 62.92 \\
Total & 69 & 20 & 89 & 100.00 \\
\hline
\end{tabular}

Table IX

Morphology Pattern of Renal Artery by sex $(n=89)$

\begin{tabular}{lcccc}
\hline Renal artery & Male & Female & Total & Percentage \\
\hline Mild Stenosis & 1 & 0 & 1 & 1.12 \\
Moderate Stenosis & 1 & 0 & 1 & 1.12 \\
Severe Stenosis & 1 & 0 & 1 & 1.12 \\
Tortuous & 0 & 0 & 0 & 0 \\
Thrombus & 0 & 0 & 0 & 0 \\
Aneurysm & 2 & 2 & 4 & 4.49 \\
Calcification & 0 & 0 & 0 & 0 \\
Normal & 64 & 18 & 82 & 92.13 \\
\hline Total & 69 & 20 & 89 & 100.00
\end{tabular}

\section{Discussion}

This retrospective observational study was done to evaluate the pattern of peripheral vascular diseases and to evaluate CT angiogram as a diagnostic tool for peripheral vascular diseases. It is the first study with CT angiogram in Bangladeshi patient. We want to show not the efficacy of the procedure but to show the pattern and severity of the peripheral vascular disease and its correlation with risk factors.

We evaluated 89 patients with clinical suspicion of PVD by CT angiogram. Most of the patients in our study were male (69 out of 89 patients). The mean age was $54.49 \pm 18.36$ yrs in male and 49.45 \pm 17.89 yrs for female, this difference is not significant. Most of the patients in our series were more than 50yrs of age (61.7\%). This also correlates with the study of Norgren et al. ${ }^{7}$
The commonest risk factor in our study was hypertension $46.1 \%$, followed by diabetes $30.3 \%$, family history of atherosclerotic coronary and peripheral vascular diseases $27 \%$, smoking $23.6 \%$, dyslipidaemia $13.5 \%$. In a prospective study Price at el have shown that the combined effect of smoking on the cardiovascular risk factor has influence on peripheral arterial disease. ${ }^{8}$ Explanation for the effect of cigarette smoking on the development of peripheral arterial disease have been described. ${ }^{9,10}$ This may reflect the gender difference in this series as none of the female subject in this study had history of smoking. Such mechanisms could include a direct toxic effect of whole smoke, nicotine and/or carbon monoxide on endothelial cells, increased platelet reactivity and agreeability, and/or a detrimental effect of the elevated white blood cell count found consistently in smokers. ${ }^{11}$ Diabetes 
mellitus was also shown as a major risk factor for atherosclerotic peripheral arterial disease. Other risk factors associated with PVD in this study also correlates with other studies. ${ }^{12}$

Abdominal aorta was mostly affected in the studied population (58.43\%). Lower limb (37.08\%), Carotid (22.47\%), Renal (7.87\%) and Upper limb arteries were affected according to decreasing frequency. Involvements of the peripheral arteries do not vary significantly in both sexes. Stenosis (5.61\%) was the predominate lesion followed by haematoma (4.49\%), Arterio-venous malformation (4.49\%) As atherosclerosis is the main pathogenesis of the peripheral vascular disease, according to the observation, morphological prevalence of lesions can be done from different arterial segments.

\section{Study Limitation}

The major drawback of CTA is large amount of intravenous contrast and the ionizing radiation involved. Also, no information is obtained regarding the flow direction and velocity. In addition, CTA may fail to demonstrate short segment stenosis, apart from the fact that horizontally oriented branches are poorly visualized, thus significant lesions may be missed in certain instances. Total number of patients in this study was also small. Despite these limitations, the vast majority of examinations we performed were considered sufficiently diagnostic to avoid more invasive imaging.

\section{Conclusion:}

Our initial experience of CT angiography with MSCT has shown that it is a promising new, fast and non-invasive imaging modality that can be utilized effectively in the evaluation of peripheral vasculature. Some of the inherent limitations of the technique and the time consumed in postprocessing can be overcome with future workstation and technology advances. Thus it would be appropriate to conclude that CTA is clearly emerging as a screening tool in patients of peripheral vascular disease. $\mathrm{CT}$ angiography is a noninvasive technique for the imaging of peripheral vascular disease. Since no data is available from a well-designed study in PVD in our country, till then the data obtained from this study can be used in
Bangladesh. As the software of CT angiogram is developing day by day, in near future at least in some cases it will replace the invasive angiogram.

Conflict of Interest - None.

\section{References:}

1. Creager M A, Libby P. Peripheral Artery Disease. Libby P, Bonow RO, Mann D L, Zipes D P, Braunwald E. editors. Braunwald Heart Disease. A textbook of Cardiovascular Medicine.2008:1491-1514

2. Hiatt W R, Medical management of peripheral Arterial Disease and claudication. N Eng J Med. 2001; 344: 16081621

3. Bloomfield P,Bradbury A,Grubb NR,Newby DE. Cardiovascular disease. In: Boon NA, Colledge NR, Walker RB. Eds. Davidson's Principle and practice of Medicine. 2006 : 601-610

4. Wennberg PW, Rooke T W. Diagnosis and Management of Diseases of The paeripheral arteries and vein.Fauster V,Alexander R W . O'Rourke RA et al . eds. Hurst's The heart.USA 2004: 2361-2379

5. Murabitto JM,D;Agastino RB,Silbertzharst H,Wilson WF. Intermittent claudication. A risk profile from The Framingham Heart Study. Circulation 1997;95: 44-49

6. Ofer A, Nitecki SS, Linn S et al. Mutidetector CT angiography of peripheral of peripheral vascular disease: a prospective comparision with intraarterial digital subtraction angiography. Am J Roentgenol 2003; 180(3) : $719-24$

7. Norgren L, Hiatt WR, Dormandy JA et al. Intersociety consensus for the management of Peripheral arterial Disease. Eur J Vasc Endovasc Surg 2007; 33: S1-S75

8. Price JF, Mowbray PI, Lee AJ, Rumleyt A, Lowe GDO, Fowkers FGR. Relationship between smoking and cardiovascular risk factors in the development of peripheral arterial disease and coronary artery disease. Eur Heart J 1999; 20:344-353.

9. Fowkes FGR. Aetiology of peripheral atherosclerosis: smoking seems especially important. $\mathrm{Br}$ Med J 1989; 298: 405-6.

10. Powell JT. Smoking. In: Fowkes FGR, ed. Epidemiology of Peripheral Vascular Disease. Berlin: Springer-Verlag, 1991:141-53.

11. Friedeman GD, Siegelaub AB, Seltzer CC, Feldman R, Collen MF. Smoking habits and the leukocyte count. Arch Environ Health 1973; 26: 137-43.

12. Al-Delaimy WK, Merchant AT, Rimm EB, Willett WC, Stampfer MJ, Hu FB. Effect of type 2 diabetes and its duration on the risk of peripheral arterial disease among men. Am J Med 2004; 116:236-40. 